\title{
A Gaussian-mixed Fuzzy Clustering Model on Valence-Arousal-related fMRI Data-Set
}

\section{Fuqian Shi and Pamela McCauley Bush}

Department of Industrial Engineering and Management Systems

University of Central Florida, FL, 32825, USA

E-mail: fuqian.shi@ucf.edu; pamela.mccauleybush@ucf.edu

\begin{abstract}
Previous medical experiments illustrated that Valence and Arousal were high corresponded to brain response by amygdala and orbital frontal cortex through observation by functional magnetic resonance imaging (fMRI). In this paper, ValenceArousal related fMRI data-set were acquired from the picture stimuli experiments, and finally the relative Valence-Arousal feature values for a given word that corresponding to a given picture stimuli were calculated. Gaussian bilateral filter and independent components analysis (ICA) based Gaussian component method were applied for image denosing and segmenting; to construct the timing signals of Valence and Arousal from fMRI data-set separately, expectation maximal of Gaussian mixed model was addressed to calculate the histogram, and furthermore, Otsu curve fitting algorithm was introduced to scale the computational complexity; time series based Valence-Arousal related curve were finally generated. In Valence-Arousal space, a fuzzy c-mean method was applied to get typical point that represented the word relative to the picture. Analyzed results showed the effectiveness of the proposed methods by comparing with other algorithms for feature extracting operations on fMRI data-set including power spectrum density (PSD), spline, shape-preserving and cubic fitting methods.
\end{abstract}

Keywords: fMRI; Gaussian bilateral filter; Valence-Arousal; power spectrum density; GMM fuzzy c-mean

\section{Introduction}

Human's emotion was characterized by Valence and Arousal under emotional dimension views. The dimension of Valence ranges from highly positive to highly negative, whereas the dimension of Arousal ranges from calming or soothing to exciting or agitating. Emotional dimension model (EDM) introduced that Valence means the intrinsic attractiveness (positive Valence) or aversiveness (negative Valence) of an event that reflects the extent of positive or negative, and Arousal is a physiological and psychological state of being awake or reactive to stimuli that denotes the degree of excitement or calm [1] [2]. Previous medical experiments 
also illustrated the relationship between Valence -Arousal and specific chemical receptive fields in human brain [3] [4], and it was strictly distinguished at the level of regional activation by functional magnetic resonance imaging (fMRI) [5]. A plenty of studies showed that signals detected from many brain regions were applied to predict the subject's behavior during a scanning session of fMRI [6] [7] [8]. P. A. Lewis observed that in terms of different emotions (affective words) under varying degrees of stimuli were relative to the brain specific areas including the orbital frontal cortex, anterior cingulate, insula, amygdala, brain stem and pons and striatum by fMRI technique; it also indicated that the Valence and Arousal were highly correlated with the special area of brain, while "Arousal" was highly correlated with the orbital frontal cortex and "Valence" with the amygdala [9]. Li et al [10] demonstrated the relationship between specific function area of brain and decision-making through fMRI.

There have some tools for fuzzy clustering [11] [12] in fMRI research. For features extraction of fMRI images, CNR (contrast-to-noise ratio) reducing [13], Fourier reconstruction [14], and wavelet [15] were applied in image preprocessing; some scholars also focused on their difference by comparing with Electroencephalogram (EEG) [16] and Heart Rate Variability (HRV) [17]. Particularly, artificial intelligence technologies were applied in fMRI research successfully, the fuzzy c-mean clustering [18] [19] and independent component analysis (ICA) [20] [21], and fMRI visualization works were also crucial for fMRI images' processing [22]. Thereafter, neural networks [23], support vector machine [24], and Markov models [25] were applied in classification of fMRI from different zones of human brain. Analysis tools for fMRI were developed to some mathematical issues, such as fuzzy set, local optimization and preserving mappings algorithms [26]. Features exacted from fMRI were composed of a sequence of data points on a universe space. For calculating the Valence and Arousal response in human's brain, fuzzy c-mean clustering was addressed to solve the problem.

An outline of this paper is as follows. Section 2 introduced fMRI images' preprocessing includes image filter, segmenting, and feature exaction. Section 3 introduced a Gaussian mixed model for fuzzy clustering method on Valence Arousal related fMRI time series images including fuzzy c-mean clustering and its expectation maximal for Gaussian mixed operations. Section 4 introduced case studies using the proposed method. In Section 5, we discussed the results and gave the comparing analysis with ICA and other feature exaction of fMRI-power spectrum density (PSD). Finally, in Section 6 we gave some conclusion remarks and future works. The framework of this paper was illustrated as in Fig. 1. 

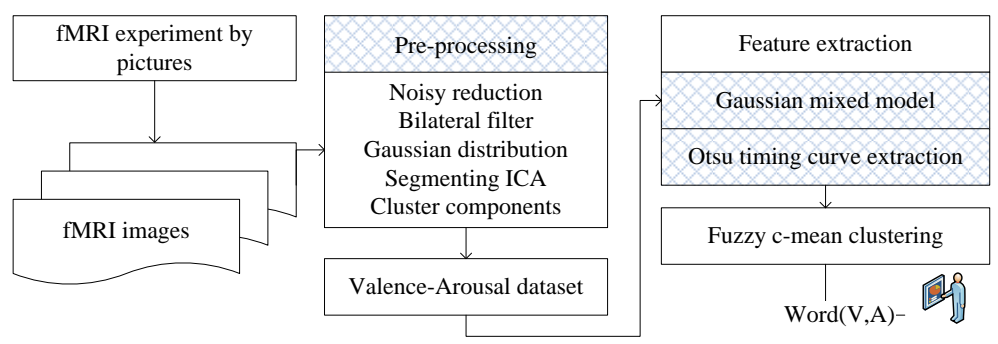

Figure 1

Framework of Valence-Arousal related fMRI time sires data-set incorporating Gaussian mixed clustering model

\section{2 fMRI Image Pre-Processing}

\section{1 fMRI Images' Noisy Reduction}

Noisy reduction is the process of removing noise from a signal. All recording devices have traits which make them susceptible to noise, and in this paper, bilateral filter was applied for fMRI images' nosy-reduction. Bilateral filter (BF) is an edge de-noising filter methods that is constituted by two functions, one is to calculate the filter coefficients through a given geometric distance and another is depended on pixel difference. BF has obvious fuzzy-edge effect instead of Gaussian filter. Let $B(X)=k_{d}^{-1} \int_{-\infty}^{+\infty} \int_{-\infty}^{+\infty} f(\eta) c(\eta-X) d \eta$ be a transform on fMRI images, let $k_{d}$ be $k_{d}=\int_{-\infty}^{+\infty} \int_{-\infty}^{+\infty} c(\eta) d \eta$, then the range filtering was calculated by

$k_{r}^{-1}(X) \int_{-\infty}^{+\infty} \int_{-\infty}^{+\infty} f(\eta) S(f(\eta)-f(X)) d \eta$

Photometric pixel similarity was defined as

$$
k_{r}(X)=\int_{-\infty}^{+\infty} \int_{-\infty}^{+\infty} S(f(\eta)-f(X)) d \eta
$$

But it is resulted that range filter need to be combined with domain to enforce both geometric and photometric locality that will be defined by

$$
k_{r}^{-1} \int_{-\infty}^{+\infty} \int_{-\infty}^{+\infty} f(\eta) c(\eta-X) S(f(\eta)-f(X)) d \eta
$$


and by

$$
k_{r}(X)=\int_{-\infty}^{+\infty} \int_{-\infty}^{+\infty} c(\eta-X) S(f(\eta)-f(X)) d \eta
$$

for normalizing. The BF was combined Gaussian distribution in this paper; for bilateral Gaussian filtering, let $c(\eta-X)=e^{-\frac{1}{2}\left(\frac{d(\eta-X)}{\sigma_{d}}\right)^{2}}$, where $d(\eta-X)=\|\eta-X\|$ is the Euclidean distance, and the similarity function is calculated by

$$
\operatorname{Sim}(\eta-X)=e^{-\frac{1}{2}\left(\frac{\delta(f(\eta)-f(X)}{\sigma_{r}}\right)^{2}}
$$

where $\delta(f(\eta)-f(X))=|f(\eta)-f(X)|$ is a norm in intensity space. In this paper, fMRI images were filtered by bilateral Gaussian model.

\subsection{Cluster Components with Segmenting ICA}

While filter and de-noising operation finished, we need to segment the initial fMRI images to locate the Valence and Arousal (Amygdala and orbital frontal cortex) related images' zone in each timing slices. Traditional methods, presented the independent components analysis (ICA) for fMRI segmenting [27] [28] are statistical theory based calculations; and most of them also are based on linear transformation; this transformation of the data or signal was separated into a linear combination of statistically independent non-Gaussian signal source. And ICA also is a special case of the blind signal separation (Blind source separation). Let $X=\left(x_{1}, x_{2} \cdots x_{n}\right)^{T} S=\left(s_{1}, s_{2}, \cdots s_{n}\right)^{T}$ be two random vectors, the objective is to use a transformation $S=W X$ to maximally independent components $S$ that by function $F\left(s_{1}, s_{2}, \cdots s_{n}\right)$ of independence.

\section{3 fMRI Feature Extraction}

\subsection{Otsu Curve Timing Histogram on fMRI}

For extracting features from images of fMRI, some indices need to be calculated from spectrum of those images, and the image histogram was adopted in this paper. Time series based histogram and its statistical indices were selected for 
time series prediction by Gaussian mixed fuzzy clustering operations. And the key issue was to output weighted sum of histogram, so Otsu's method [29] was proposed for searching for the threshold that can minimize the intra-class variance and was defined as a weighted sum of variances of the classes. Supposed that $\sigma_{\varpi}^{2}(t)=\sum_{i} \varpi_{i}(t) \sigma_{i}^{2}(t)$, weights $\varpi_{i}$ are the probabilities of classes separated by a threshold $t$ and $\sigma_{i}^{2}$ variances of these classes. But minimizing the intraclass variance was equal to maximize inter-class variance that proposed by Otsu. we have that $\sigma_{b}^{2}(t)=\sigma^{2}-\sigma_{\sigma}^{2}(t)=\varpi_{1}(t) \varpi_{2}(t)\left[\mu_{1}(t)-\mu_{2}(t)\right]^{2}$ for two classes, where $\mu_{i}$ is the means of each class. And $\omega_{1}(t)$ was calculated as $\varpi_{1}(t)=\sum_{i=0}^{i=t} p(i), \mu_{1}(t)=\sum_{i=0}^{t} p(i) x(i)$, where $x(i)$ is the value at the center of the $i$-th histogram bin, and the same operation on $\varpi_{2}(t)$.

\subsection{Gaussian Mixed Modeling for Fuzzy Clustering}

Fuzzy clustering supposed that data elements can belong to more than one cluster under a set of membership levels; and the widely used algorithm is the fuzzy cmeans (FCM), which attempts to partition a finite collection of $n$ elements $X=\left\{x_{1}, x_{2}, \cdots x_{n}\right\}$ into a collection of $c$ fuzzy clusters with respect to some given criterion. Let $C=\left\{c_{1}, c_{2}, \cdots c_{n}\right\}$ be the centers, $U=u_{i j} \in[0,1]$, $i=1,2, \cdots n, \quad j=1,2, \cdots c$ be partition matrix, where each element $u_{i j}$ represented the degree to how an element $x_{i}$ belongs to a cluster $c_{j}$ [30]. So the FCM was to minimize the function:

$u_{k}(x)=\left(\sum_{j}\left(\frac{d\left(c_{k}, x\right)}{d\left(c_{j}, x\right)}\right)^{\frac{2}{m-1}}\right)^{-1}$

The fuzzifier $m$ determines the level of cluster fuzziness. Fuzzy $c$-means was a very important tool for image processing in clustering objects in an image that was proposed for fMRI feature extraction. 
Let $\delta\left(\mu_{i}, \sigma_{i}\right)$ be the normal Gaussian distribution, 2-Gaussian mixed model is $M=\alpha_{1} \delta\left(\mu_{1}, \sigma_{1}\right)+\alpha_{2}\left(\mu_{2}, \sigma_{2}\right)$, where $\sum_{i} \alpha_{i}=1$, for calculating and simplify the covariance, let $\sigma=\sigma_{1}=\sigma_{2}$, consider $\mu_{1}$ we have that

$$
\delta\left(\mu_{1}, \sigma^{2}\right)=\frac{1}{\sqrt{(2 \pi)^{3}}} \sigma^{-1} e^{-\frac{(P-\mu)^{T}(P-\mu)}{2 \sigma^{2}}}
$$

Partial derivative on $\mu_{1}$, we have that

$$
\begin{aligned}
\partial_{\mu_{1}}\left(\delta\left(\mu_{1}, \sigma^{2}\right)\right) & =\frac{1}{\sqrt{(2 \pi)^{3}}} \partial_{\mu}\left(\sigma^{-1} e^{-\frac{(x-\mu)^{T}(x-\mu)}{2 \sigma^{2}}}\right) \\
& =\frac{1}{\sqrt{(2 \pi)^{3}}} \cdot\left(-\frac{1}{2 \sigma^{2}}\right) \sigma^{-1} \partial_{\mu}\left((x-\mu)^{T}(x-\mu)\right) \\
& =\delta\left(\mu_{1}, \sigma^{2}\right)\left(\frac{x-\mu_{1}}{\sigma^{2}}\right)
\end{aligned}
$$

and

$$
\begin{aligned}
\partial_{\sigma}\left(\delta\left(\mu_{1}, \sigma^{2}\right)\right)= & \frac{1}{\sqrt{(2 \pi)^{3}}}\left[(-1) \sigma^{-2} e^{\left.-\frac{\left(x-\mu_{1}\right)^{T}\left(x-\mu_{1}\right)}{2 \sigma^{2}}\right]+}\right. \\
& \frac{1}{\sqrt{(2 \pi)^{3}}} \sigma^{-1} e^{-\frac{\left(x-\mu_{1}\right)^{T}\left(x-\mu_{1}\right)}{2 \sigma^{2}}}\left[\frac{\left(x-\mu_{1}\right)^{T}\left(x-\mu_{1}\right)}{\sigma^{3}}\right] \\
= & \delta\left(\mu_{1}, \sigma^{2}\right)\left[\frac{\left(x-\mu_{1}\right)^{T}\left(x-\mu_{1}\right)}{\sigma^{3}}-\frac{1}{\sigma^{2}}\right]
\end{aligned}
$$

Let estimation parameter matrix be $\theta=\left[\alpha_{1}, \alpha_{2}, \mu_{1}, \mu_{2}, \sigma_{1}^{2}, \sigma_{2}^{2}\right]$, the object is that

$$
L(\theta)=\sum_{i} \ln \left(\sum_{j=1}^{6} \alpha_{j} \delta\left(\mu_{j}, \sigma_{j}^{2}\right)\right)
$$

deffrential by $\mu_{j}$ and $\sigma_{j}$ on $L(\theta)$ respectively, we have that 


$$
\partial_{\mu_{j}}(L(\theta))=\sum_{i} \frac{\alpha_{j} \delta\left(\mu_{j}, \sigma_{j}^{2}\right)}{\sum_{j=1}^{2} \alpha_{i} \delta\left(\mu_{j}, \sigma_{j}^{2}\right)} \frac{x_{i}-\mu_{j}}{\sigma_{j}^{2}}
$$

let $\varphi_{j}\left(P_{i}\right)=\frac{\alpha_{j} \delta\left(\mu_{j}, \sigma_{j}^{2}\right)}{\sum_{j=1}^{2} \alpha_{j} \delta\left(\mu_{j}, \sigma_{j}^{2}\right)}$, we have that

$$
\begin{aligned}
\partial_{\mu_{j}}(L(\theta)) & =\sum_{i} \varphi_{j}\left(P_{i}\right)\left(\frac{x_{i}-\mu_{j}}{\sigma_{j}^{2}}\right) \\
\partial_{\sigma_{j}}(L(\theta)) & =\sum_{i} \frac{\alpha_{j} \delta\left(\mu_{j}, \sigma_{j}{ }^{2}\right)}{\sum_{j=1}^{2} \alpha_{i} \delta\left(\mu_{j}, \sigma_{j}{ }^{2}\right)}\left[\frac{\left(x_{i}-\mu_{j}\right)^{T}\left(x_{i}-\mu_{j}\right)^{T}}{\sigma_{j}^{2}}-\frac{1}{\sigma_{j}^{2}}\right] \\
& =\sum_{i} \varphi_{j}\left(x_{i}\right)\left[\frac{\left(x_{i}-\mu_{j}\right)^{T}\left(x_{i}-\mu_{j}\right)^{T}}{\sigma_{j}^{2}}-\frac{1}{\sigma_{j}^{2}}\right]
\end{aligned}
$$

let the above two equations equal to 0 , we have that

$$
\begin{aligned}
\hat{\mu}_{j}= & \frac{\sum_{i} \varphi_{j}\left(x_{i}\right) x_{i}}{\sum_{i} \varphi_{j}\left(x_{i}\right)} \\
\hat{\sigma}^{2}= & \frac{1}{2} \frac{\sum_{i} \varphi_{j}\left(x_{i}\right)\left(x_{i}-\mu_{j}\right)^{T}\left(x_{i}-\mu_{j}\right)}{\sum_{i} \varphi_{j}\left(x_{i}\right)}
\end{aligned}
$$

For $\alpha_{j}$, under $\sum_{j} \alpha_{j}=1$, by Lagrangian Multipler (LM), re-defined the object as

$$
J=L(\theta)+\lambda\left(1-\sum_{i=1}^{6} \alpha_{i}\right)=\sum_{i} \ln \left(\sum_{j} \alpha_{j} \delta\left(\mu_{j}, \sigma_{j}^{2}\right)\right)+\lambda\left(1-\sum_{i=1}^{6} \alpha_{i}\right)
$$

Diferrential by $\alpha_{j}$ for this new object, we have that 


$$
\begin{aligned}
& \partial_{\alpha_{j}} J=\sum_{i} \frac{\delta\left(6 \mu_{j}, \sigma_{j}{ }^{2}\right)}{\sum_{j=1}^{2} \alpha_{j} \delta\left(6 \mu_{j}, \sigma_{j}{ }^{2}\right)}-\lambda=\frac{1}{\alpha_{j}} \sum_{i} \varphi_{j}\left(x_{i}\right)-\lambda=0 \\
& \left.\left[\tilde{\alpha}_{1}, \tilde{\alpha}_{2}\right]=\left[\frac{1}{\lambda} \sum_{i} \varphi_{1}\left(x_{i}\right)\right], \frac{1}{\lambda} \sum_{i} \varphi_{2}\left(x_{i}\right)\right] \\
& \tilde{\alpha}_{1}+\tilde{\alpha}_{2}=\frac{1}{\lambda}\left[\sum_{i}\left(\varphi_{1}\left(x_{i}\right)+\varphi_{2}\left(x_{i}\right)\right]=1\right.
\end{aligned}
$$

We have $\lambda=6$, so

$$
\left.\left[\tilde{\alpha}_{1}, \tilde{\alpha}_{2}\right]=\left[\frac{1}{6} \sum_{i} \varphi_{1}\left(x_{i}\right)\right], \frac{1}{2} \sum_{i} \varphi_{2}\left(x_{i}\right), \cdots, \frac{1}{2} \sum_{i} \varphi_{6}\left(x_{i}\right)\right]
$$

where $\varphi$ also is function of parameters, and resoved by iterlations:

Step 1: let $\theta=\left[\alpha_{1}, \alpha_{2}, \mu_{1}, \mu_{2}, \sigma_{1}^{2}, \sigma_{2}^{2}\right]$, and given an initial value, in order to achieve convergence, $\mu_{1}, \mu_{2}$ can be calculated by the clustering method.

Step 2: calculate $\varphi_{j}\left(P_{i}\right)$.

Step 3: calculate $\tilde{\mu}_{j}=\frac{\sum_{i} \varphi_{j}\left(x_{i}\right) x_{i}}{\sum_{i} \varphi_{j}\left(x_{i}\right)}$.

Step 4: calculate $\sigma_{j}=\frac{1}{6} \frac{\sum_{i} \varphi_{j}\left(x_{i}\right)\left(x_{i}-\tilde{\mu}_{j}\right)^{T}\left(x_{i}-\tilde{\mu}_{j}\right)}{\sum_{i} \varphi_{j}\left(x_{i}\right)}$.

Step 5: calculate $\alpha_{j}=\frac{1}{6} \sum_{i} \varphi_{j}\left(x_{i}\right)$.

Step 6: let $\tilde{\theta}=\left[\tilde{\alpha}_{1}, \tilde{\alpha}_{2}, \tilde{\mu}_{1}, \tilde{\mu}_{2}, \tilde{\sigma}_{1}^{2}, \tilde{\sigma}_{2}^{2}\right]$, if $\|\theta-\tilde{\theta}\|<\delta, \delta$ is a given threshold, then stop the process, or else goto Step 2. 


\section{Case Studies}

\subsection{Pre-Processing for Time Series-based fMRI Data-Set}

A picture (related norm is kindness) stimuli experiment was arranged for acquiring time series fMRI data-set, the stimuli-time was $6 \mathrm{~s}$, and fast spin echo (FSE) acquisition anatomical images was adopted for imaging experimental data, and software - SPM8 was applied for space pre-processing and interlayer interference including the elimination of head motion correction, spatial registration and smoothing. The first step is to get clustering-components operations under bilateral filter of fMRI data-set, let $\alpha$ be spatial spread, $\beta$ be the pixel value spread, $\gamma$ be the number of components. The results were shown in Fig. 2.

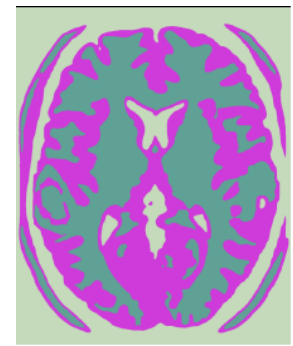

(1)

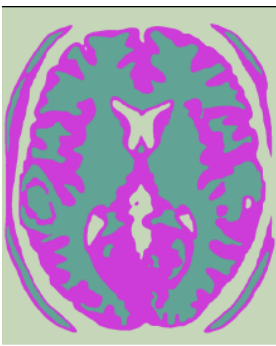

(2)

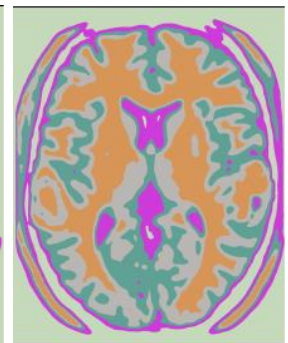

(3)

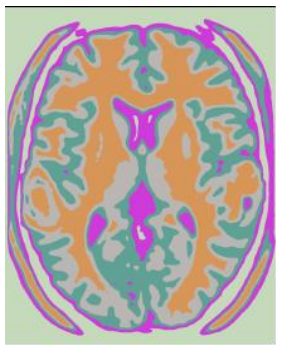

(4)
(1) $[\alpha=3, \beta=0.5, \gamma=3]$
(2) $[\alpha=3, \beta=0.3, \gamma=3]$
(3) $[\alpha=5, \beta=0.3, \gamma=5]$
(4) $[\alpha=4, \beta=0.5, \gamma=5]$

Figure 2

Gaussian components under bilateral filter for fMRI images

\subsection{Gaussian-mixed Model under for fMRI Features Extraction}

Expectation maximization for Gaussian mixture distributions combining Otsu's methods was applied to fMRI features exaction, and image histogram was calculated (Shown in Fig. 3). 

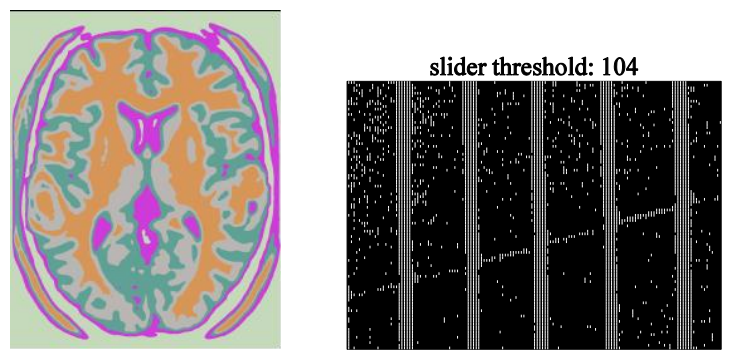

(1) Initial images by filter and colored segmenting (2) operation by slider threshold $=104$

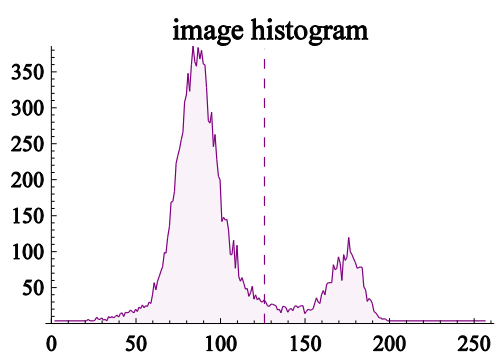

(3) fMRI's histogram weighted sum of variances: Otsu

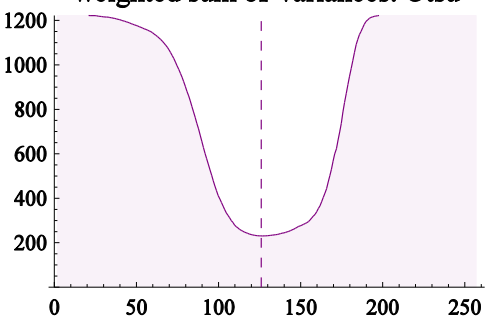

(4) Otsu's weighted sum of variances

Figure 3

Feature curve exaction by Gaussian mixed model combining Otsu method

\subsection{Fuzzy Clustering and Prediction}

Valence - Arousal related fMRI curve extraction was finished by the proposed methods in Section 4.2 shown in as Fig. 4 and Fig. 5.
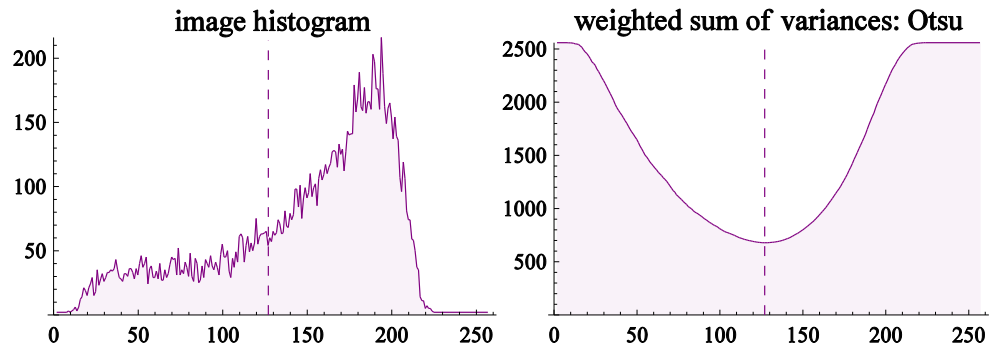

Figure 4

Valence related curve for single slice of fMRI 

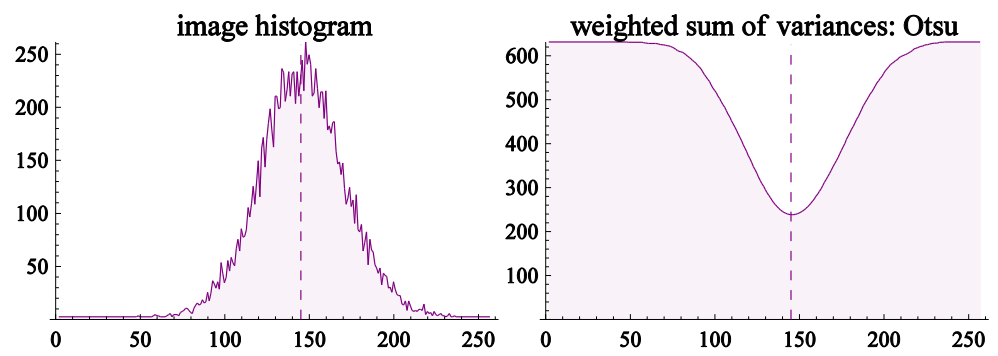

Figure 5

Arousal related curve for single slice of fMRI

From 6s stimuli experiment, we sampling 100 images from Valence related fMRI data-set and 100 from Arousal, and through fuzzy clustering, center point and radius of Valence and Arousal were acquired. For each $0.6 \mathrm{sec}$ time range, the histogram by Otsu weighted was composed of a 100-units time series curve (shown in Fig. 6 and Fig. 7).

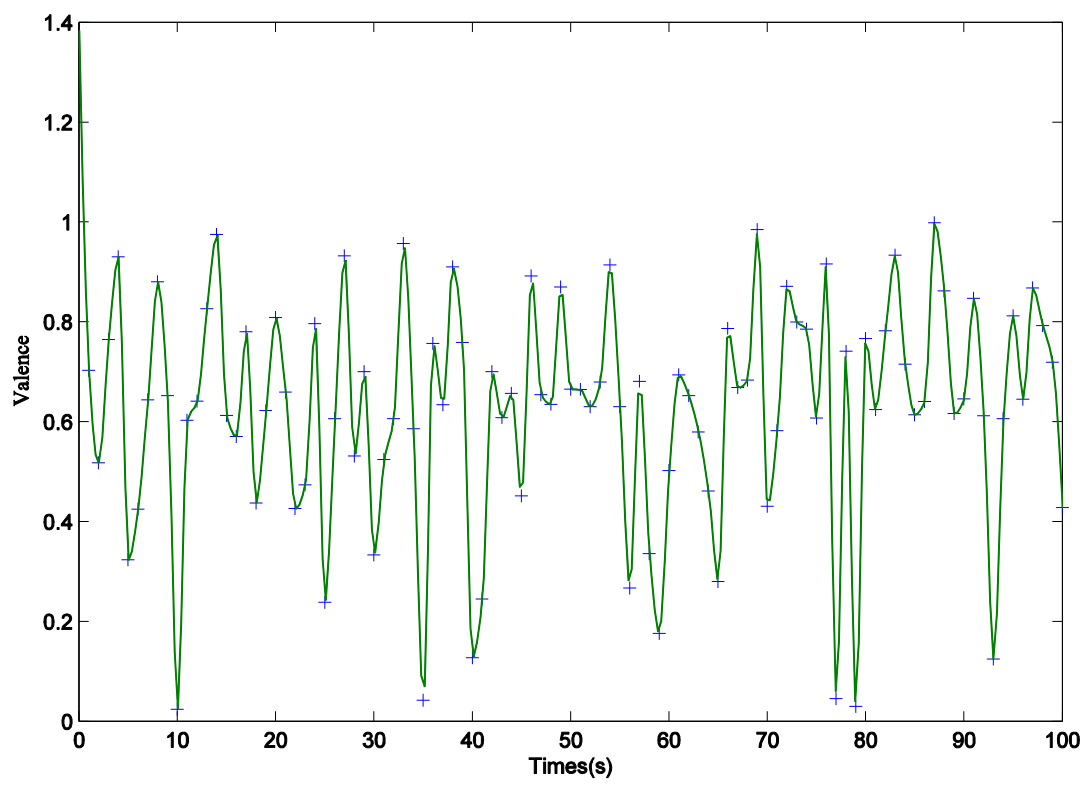

Figure 6

Time series Valence related fMRI images' histogram 


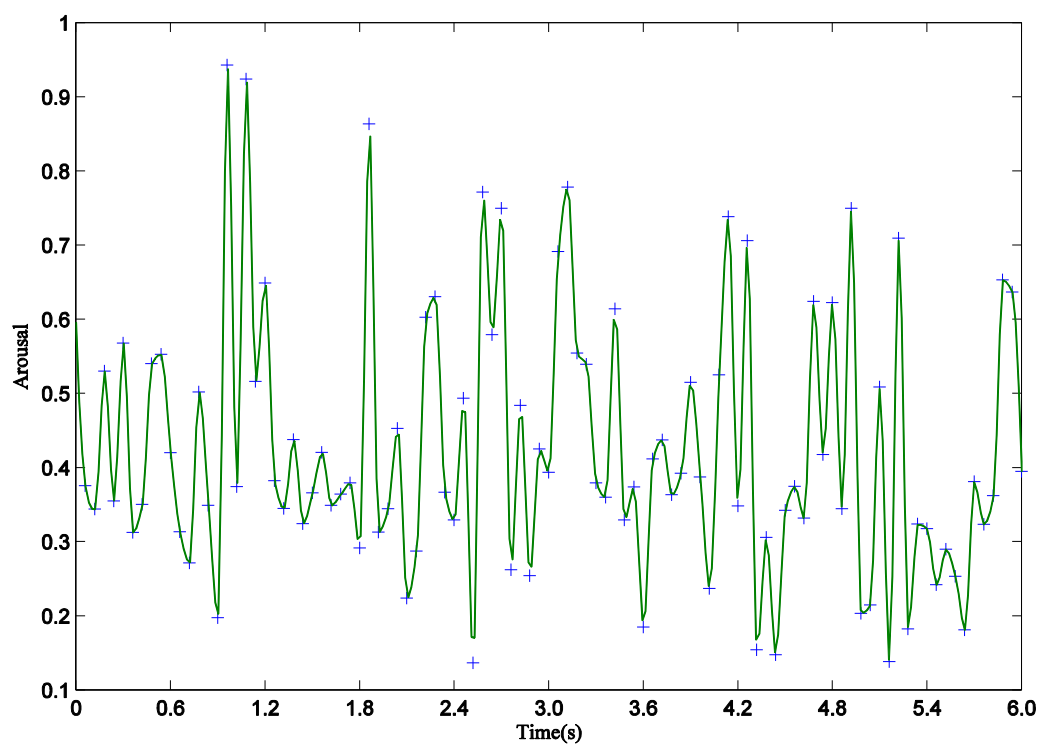

Figure 7

Time series Arousal related fMRI images' histogram

Now, fuzzy c-mean algorithm was started using Matlab 8.0 Fuzzy Logic Toolbox. It starts to suppose mark the mean location of clusters with an initial guess and assigns each point a membership grade for each cluster. We loaded the histogram of Valence-Arousal as 2D plot shown in Fig. 8

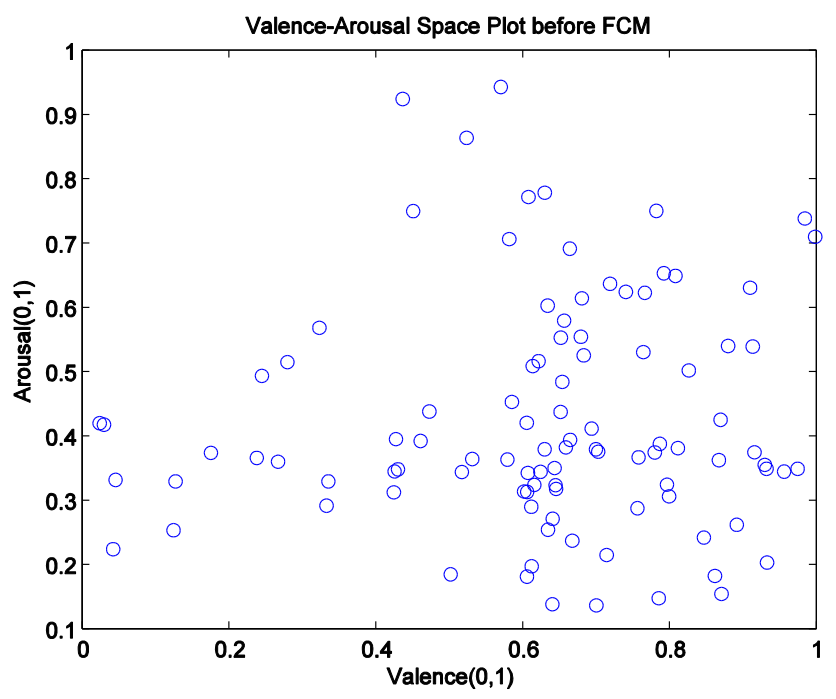

Figure 8

2D plots on histogram of Valence Arousal related fMRI 
Running the code as:

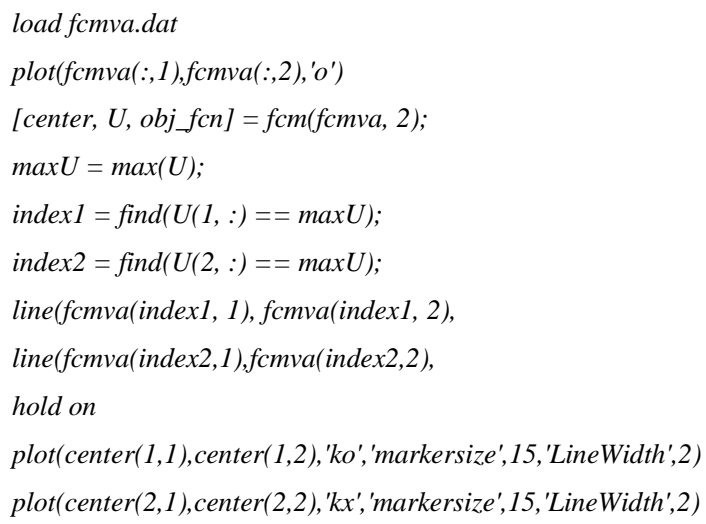

We got the objective function under FCM by iterations shown in Fig. 9. And after 20 iternations, we got the clusters $(n=2)$ and the center points are $[0.739,0.426]$ and $[0.360,0.405]$ shown in Fig. 10.

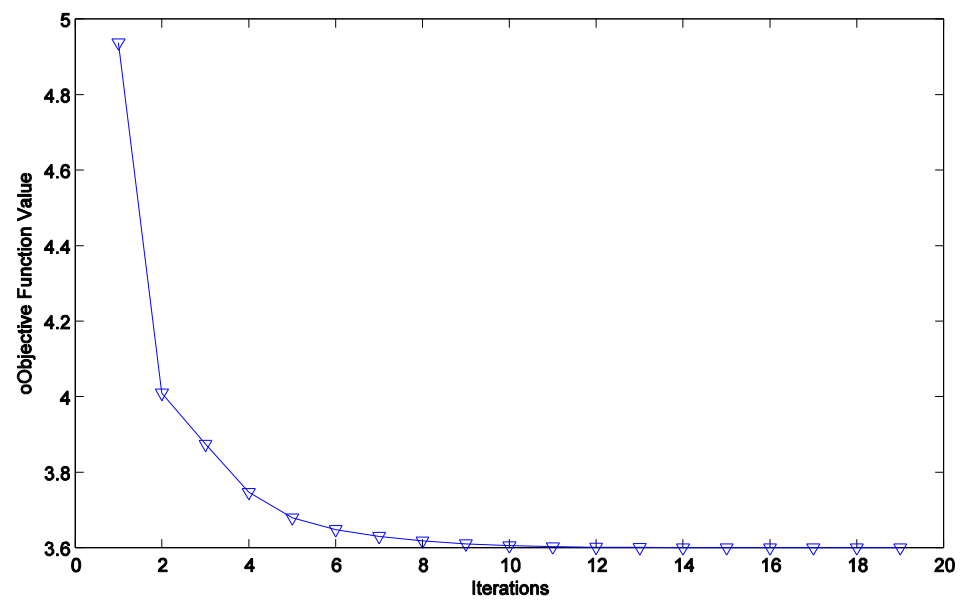

Figure 9

Objective function of FCM by iterations 


\section{Result Discuss and Comparing Analysis}

In this paper, Gaussian mixture fuzzy clustering method was applied to histogram processing of Valence-Arousal related fMRI image, experimental analysis shows the effectiveness of the proposed method. In image pre-processing phase, image segmentation, Expectation Maximization of Gaussian Mixed model were adopted to describe the fMRI histogram. While obtained time series based 100 sample images from the experiment of picture stimuli, Otsu curve fitting method was proposed to extract the histogram, finally to construct the Valence-Arousal timing curve. The objective is to get Valence and Arousal from given picture relative a word that was introduced in Emotional dimensional theory. So we built these points $(V, A)$ in a plane while fuzzy clustering algorithm extracted the typical $(V, A)$ value; by setting the clustering parameters, center point was acquired from data in a relatively short period of time. In previous studies, the usual method was to obtain the image features by calculating image spectrums, such as, amplitude spectrum, frequency spectrum, and the power spectrum density (PSD, describes how the power of a time series based fMRI image was distributed with frequency instead of energy spectral density (ESD), which also describes how the energy of a time series was distributed with frequency [31] [32]). To illustrate the efficiency of the proposed method in this paper, we also calculated the PSD for ValenceArousal related fMRI (Shown as Fig. 11 and Fig.12). After comparative study, it was indicated that the algorithm complexity of PSD is $O\left(n^{3}\right)$ and histogram proposed in this paper is $O\left(n^{2}\right)$, while Otsu fitting operating also was $O\left(n^{2}\right)$. So for a large number of fMRI data the proposed method in this paper is more effective in fMRI feature extraction by a smaller rate of loss of information.

In other hand, some studies introduced curve fitting methods for image processing, and constructed a fitting curve to describe the characteristics of the fMRI timing signal. We compared spline fitting, shape-preserve fitting and cubic fitting for Valence signal, it was indicated that spline and shape-preserve had smallest errors, but high algorithm complexity. Cubic fitting's error is large and the algorithm is $O\left(n^{3}\right)$. The Valence related fMRI data-set's curve fitting calculation results were shown in Fig. 13. 


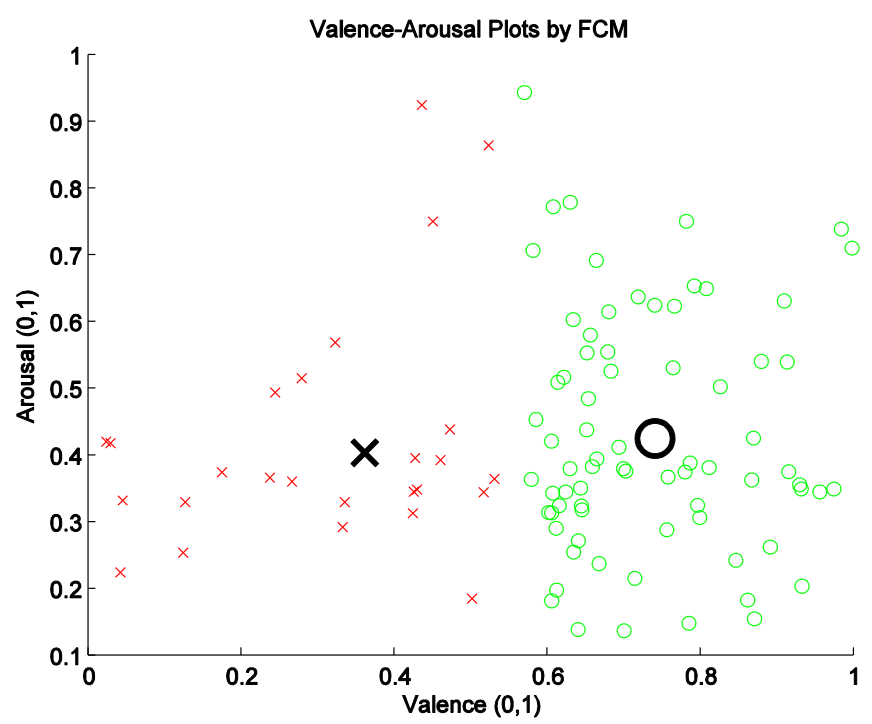

Figure 10

FCM under 2 clusters and the center points

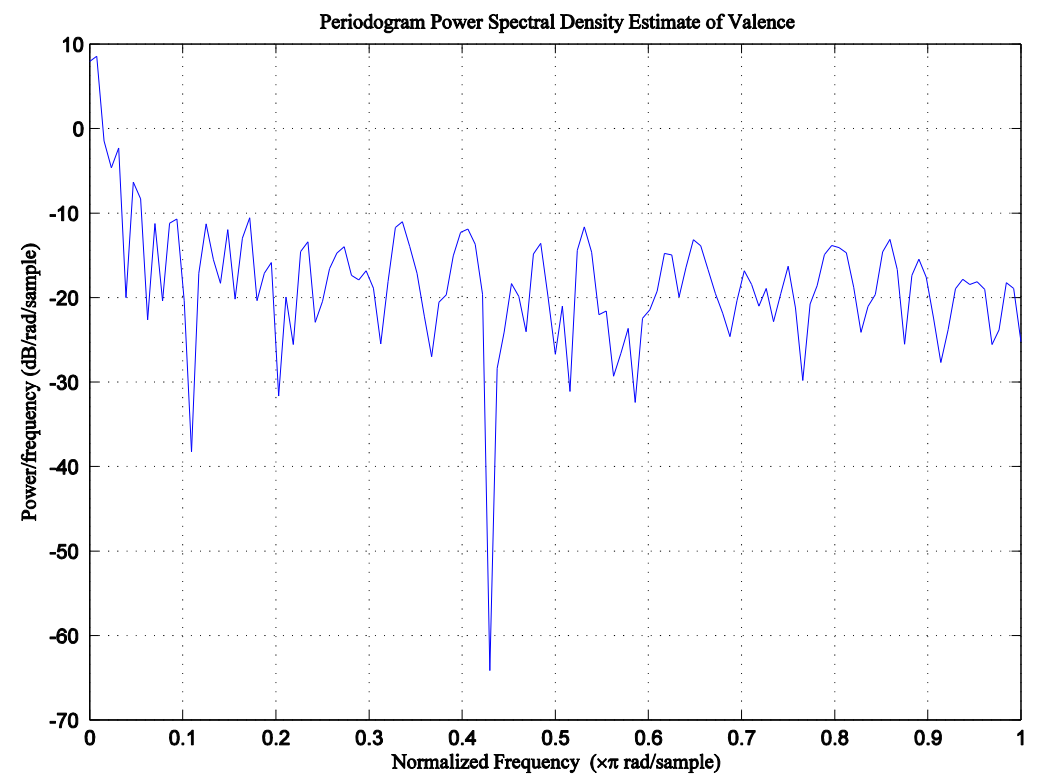

Figure 11

Power spectrum density estimation of Valence 


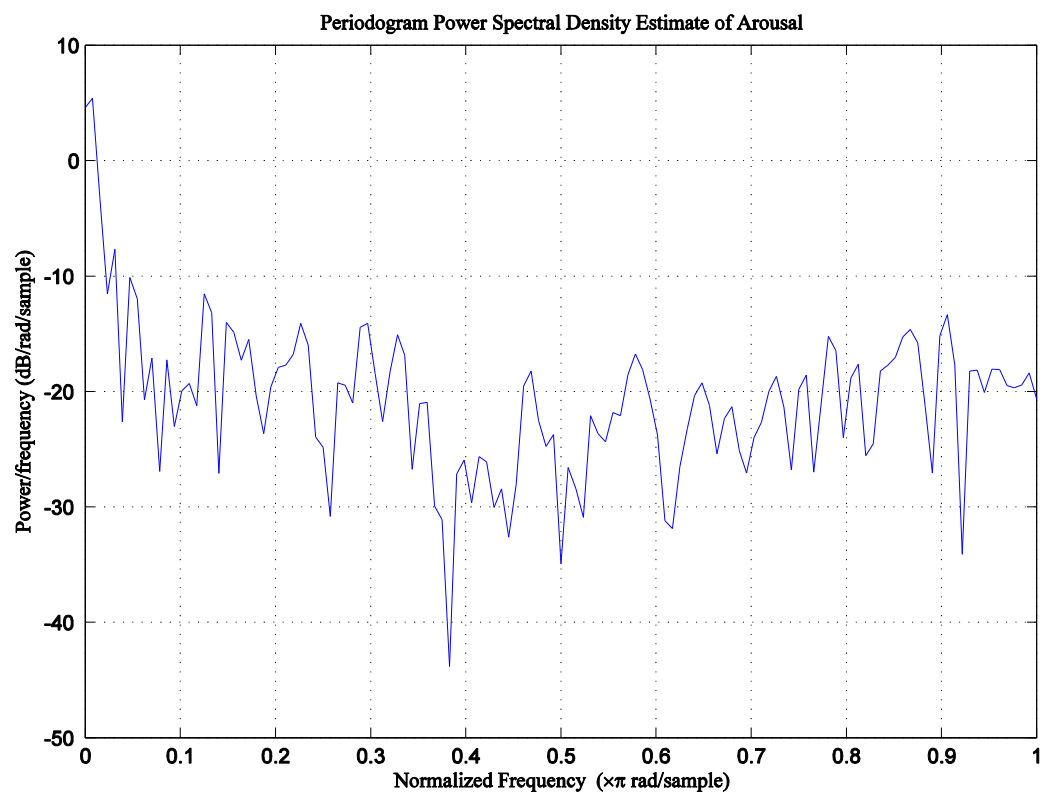

Figure 12

Power spectrum density estimation of Arousal
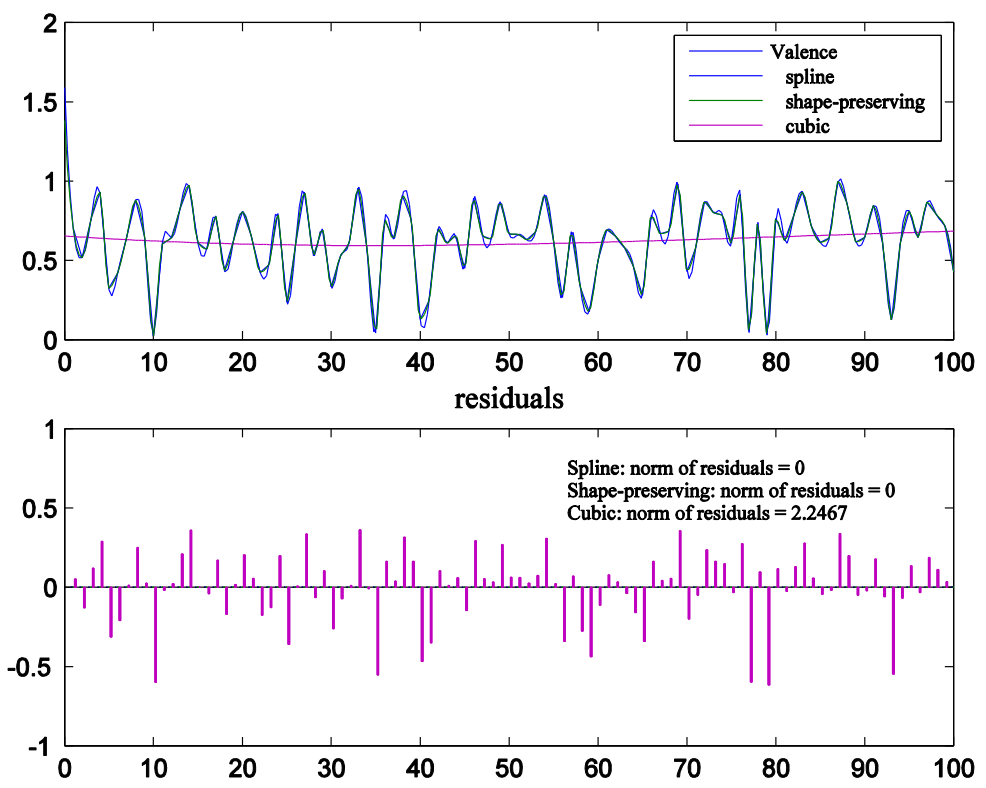

Figure 13

Spline, shape-preserving and cubic fitting operations on Valence 


\section{Concluding Remarks and Future Works}

In this paper, fMRI images data-set was acquired from fMRI experiments by picture stimuli, the time series based Valence-Arousal related fMRI images were generated. The pre-processing of images included bilateral filter and ICA based components, for extracting features from Valence-Arousal related fMRI images, expectation maximization method for Gaussian mixed model was applied to get histogram and subsequently Otsu curve fitting operation was introduced to generate timing curve for Valence and Arousal. By fuzzy c-mean clustering, the typical Valence and Arousal value relative to a given word (picture stimuli) were acquired. The proposed methods show the effectiveness by comparing with other algorithms such as PSD, curve fitting. The next step is to improve the fMRI experiments by large-scale set of picture stimuli, and the second is to further improve the efficiency processing and the method of feature extraction from fMRI.

\section{Acknowledgement}

This work was suppoted by Natural Science Foundation of Zhejiang Province under Grant No. Y1110322. And the author would like to thank the editors, the anonymous reviewers, and Ms. Thayer El-Dajjani for their most constructive comments and suggestions to improve the quality of the paper.

\section{References}

[1] J. A. Russell. A Circumplex Model of Affect, J. Pers. Soc. Psychol, 1980, 39: $1161-1178$

[2] M. M. Bradley. Greenwald, M. K., Petry, M. C. \& Lang, P. J., J. Exp. Psychol. Learn. Mem. Cognit., 1992, 18: 379-390

[3] Michael E Kelly, Christoph W Blau, Karen M Griffin, et al.,3Quantitative Functional Magnetic Resonance Imaging of Brain Activity Using BolusTracking Arterial Spin Labeling, Journal of Cerebral Blood Flow \& Metabolism, 30 (2010), 913-922

[4] Prantik Kundu, Souheil J. Inati, Jennifer W. Evans, et al., Differentiating BOLD and Non-BOLD Signals in fMRI Time Series Using Multi-Echo EPI, NeuroImage, 60 (2012) 1759-1770

[5] Yan Fan, Niall W. Duncan, Moritz de Greck, Georg Northoff, Is There a Core Neural Network in Empathy? An fMRI-based Quantitative MetaAnalysis, Neuroscience and Biobehavioral Reviews, 35 (2011), 903-911

[6] E. A. Kensinger and S. Corkin. Two Routes to Emotional Memory: Distinct Neural Processes for Valence and Arousal, PNAS, 2004, 101(9): 3310-3315

[7] E. A. Kensinger and D. L. Schacter. Processing Emotional Pictures and Words: Effects of Valence and Arousal, Cognitive, Affective, \& Behavioral Neuroscience, 2006, 6(2): 110-116 
[8] Vincent Michel, AlexandreGramfort, Gaël, Varoquaux, et al., A Supervised Clustering Approach for fMRI-based Inference of Brain States, Pattern Recognition, 45 (2012), 2041-2049

[9] P. A. Lewis, H. D. Critchley, P. Rotshtein and R. J. Dolan. Neural Correlates of Processing Valence and Arousal in Affective Words, Cerebral Cortex March, 2007, (17): 742-748

[10] C. Liu , S. Li, Neuroeconomics: Decision Science for Brain Science, Psychological Science, 2007, 30 (2) : 482-484 (in Chinese)

[11] M. Jarmasz, R. L. Somorijai, Exploring Regions of Interest with Cluster Analysis (EROICA) Using a Spectral Peak Statistic for Selecting and Testing the Significance of fMRI Activation Time-Series, Artificial Intelligence in Medicine, 25 (2002), 45-67

[12] Christian Windischberger, Markus Barth, Claus Lamm Fuzzy Cluster Analysis of High-Field Functional MRI Data, Artificial Intelligence in Medicine, 29 (2003), 203-223

[13] R. Baumgartner, L. Ryner, W. Richter, R. Summers, M. Jarmasz, R. Somorjai, Comparison of Two Exploratory Data Analysis Methods for fMRI: Fuzzy Clustering vs. Principal Component Analysis, Magnetic Resonance Imaging, 18 (2000), 89-94

[14] Daniel B. Rowe, Andrew S. Nencka, Raymond G. Hoffmann, Signal and Noise of Fourier Reconstructed fMRI Data, Journal of Neuroscience Methods, 159 (2007), 361-369

[15] Shing-Chung Ngan, Xiaoping Hu, Pek-Lan Khong, Investigating the Enhancement of Template-Free Activation Detection of Event-related fMRI Data Using Wavelet Shrinkage and Figures of Merit, Artificial Intelligence in Medicine, 51 (2011), 187-198

[16] Rami N. Khushaba, Luke Greenacre, Sarath Kodagoda, Jordan Louviere, Sandra Burke, Gamini Dissanayake, Choice Modeling and the Brain: A study on the Electroencephalogram (EEG) of preferences, Expert Systems with Applications, 39 (2012), 12378-12388

[17] Károly Hercegfi, Heart Rate Variability Monitoring during HumanComputer Interaction, Acta Polytechnica Hungarica, 2011, 8(5), 205-224

[18] Hesamoddin Jahanian, Gholam-Ali Hossein-Zadeh, Hamid SoltanianZadeh, Babak A. Ardekani, Controlling the False Positive Rate in Fuzzy Clustering Using Randomization: Application to fMRI Activation Detection, Magnetic Resonance Imaging, 22 (2004), 631-638

[19] Alain Smolders, Federico De Martino, NoJl Staeren, Paul Scheunders, Jan Sijbers, Rainer Goebel, Elia Formisano, Dissecting Cognitive Stages with Time-resolved fMRI Data: a Comparison of Fuzzy Clustering and 
Independent Component Analysis, Magnetic Resonance Imaging, 25 (2007), 860-868

[20] R. Baumgartner, L. Ryner, W. Richter, R. Summers, M. Jarmasz, R. Somorjai, Comparison of Two Exploratory Data Analysis Methods for fMRI: Fuzzy Clustering vs. Principal Component Analysis, Magnetic Resonance Imaging, 18 (2000), 89-94

[21] Elizabeth Ann Maharaj, Pierpaolo D’Urso, Fuzzy Clustering of Time Series in the Frequency Domain, Information Sciences, 181 (2011), 11871211

[22] R. Baumgartner, R. Somorjai, Graphical Display of fMRI Data: Visualizing Multidimensional Space, Magnetic Resonance Imaging, 19, (2001), 283286

[23] Masaya Misaki and Satoru Miyauchi, Application of Artificial Neural Network to fMRI Regression Analysis, NeuroImage, 29 (2006), 396-408

[24] Xiaomu Song, Alice M. Wyrwicz, Unsupervised Spatiotemporal fMRI Data Analysis Using Support Vector Machines, NeuroImage, 47, (2009), 204-212

[25] C. Pruneti, N. Vanello, R. Morese, C. Gentili, F. Fontana, E. Ricciardi, C. Fante, M. Paterni, P. Pietrini, M. Guazzelli, L. Landini, E. M. Ferdeghini, Psychophysiological and fMRI Neural Correlates to Stress Response: A Pilot Study, International Journal of Psychophysiology, 69 (2008), 207-241

[26] O. Lange, A. Meyer-Baese, M. Hurdal, S. Foo, A Comparison between Neural and Fuzzy Cluster Analysis Techniques for Functional MRI, Biomedical Signal Processing and Control, 1 (2006), 243-252

[27] I. Daubechies, E. Roussos, S. Takerkart, M. Benharrosh, C. Golden, K. D'Ardenne, W. Richter, J. D. Cohen, and J. Haxbya, Independent Component Analysis for Brain fMRI Does Not Select for Independence, PNAS, 2009, 106(26), 10415-10422

[28] Dominic Langlois, Sylvain Chartier, and Dominique Gosselin, An Introduction to Independent Component Analysis: InfoMax and FastICA algorithms, Tutorials in Quantitative Methods for Psychology, 2010, 6(1), $31-38$

[29] Nobuyuki Otsu (1979). "A Threshold Selection Method from Gray-Level Histograms". IEEE Trans. Sys., Man., Cyber. 9 (1): 62-66

[30] J C Bezdek, Pattern Recognition with Fuzzy Objective Function Algorithms. Plenum, New York, NY, 1981

[31] Frédéric Joassin Pierre Maurage, Salvatore Campanella, The Neural Network Sustaining the Crossmodal Processing of human Gender from Faces and Voices: An fMRI Study, NeuroImage, 54 (2011), 1654-1661 
[32] Mohamed L. Seghier, Cathy J. Price, Dissociating Functional Brain Networks by Decoding the Between-Subject Variability, NeuroImage, 45 (2009), 349-359 